\title{
Análise de conteúdo das sessões de comunicação científica do I Congresso Nacional de Psicologia Escolar
}

\author{
Carla Witter \\ Universidade São Judas Tadeu
}

\author{
Maria Terezinha C. P. Yukimitsu \\ Universidade São Judas Tadeu
}

\begin{abstract}
Summary
Esta pesquisa analisou o conteúdo das sessões de comunicação científica. Juízes: duas psicólogas e professoras de Psicologia Escolar; Material: os Anais do I Congresso Nacional de Psicologia Escolar; Procedimento: o material foi analisado independentemente pelos juízes $\left(r_{0}=0,87\right)$. Os resultados evidenciaram maior freqüência em pesquisas do tipo de levantamento; os temas concentraram-se em Psicologia Escolar; o local e os sujeitos mais investigados foram o 10 grau e o aluno; e os conteúdos destacados foram Psicologia Escolar e Aluno. Conclui-se que há necessidade de estudos mais sofisticados sobre o assunto.
\end{abstract}

Palavras-chave: Análise de conteúdo, produção científica, literatura cinzenta.

\begin{abstract}
s
These research analysed the content of cientific communicatioDS. Judges: two psychologists and teacher of School Psychology, too; Material: the Annual of I Congresso Nacional de Psicologia Escolar; Procedure: the material was analysed by judges ( $\mathrm{ro}=0,87)$. The results point out the most frequency in researchs of survey; the themes was in School Psychology; the local and subjects more investigated were the first degree and the student; and the detach contents were in School Psychology and Student. These concludes that is necessary more sofisticated studies about these matter.
\end{abstract}

Key words: content analyses, scientific production, gray literature.

A literatura científica (Eisenberg, 1991; CFP, 1992) salienta a necessidade de trabalhos que analisem a produção de conhecimento de uma determinada. área de estudo, de forma a fornecer dados a respeito da quantidade e qualidade de pesquisas e, conseqüentemente, de conhecimentos produzidos que revelem o crescimento do "saber-poder-fazer" de uma determinada área da ciência. Este tipo de pesquisa, sobre produção científica, é nomeada de metaciência, nos meios acadêmicos, por investigar o desenvolvimento da própria ciência.

Witter e colaboradores (1992), em pesquisa sobre a atuação do Psicólogo Escolar e Educacional no Brasil, destacam em suas conclusões três aspectos relevantes, quanto ao tema aqui enfocado, que são: (1) em relação aos parâmetros internacionais, a produção científica na área escolar é limitada; (2) há predominância da divulgação dos trabalhos em anais; e (3) existe carência de pesquisas.

Dentro desta perspectiva, a presente pesquisa procurou investigar a produção científica, apresentada sob a forma de comunicações, no I Congresso Nacional de Psicologia Escolar realizado no Brasil, em 1991. Para tanto, se estabeleceu por objetivo geral, analisar o conteúdo das sessões de comunicação científica; e foram estabelecidos os seguintes objetivos específicos: (1) levantar o tipo de pesquisas apresentadas pelas universidades particulares e públicas; (2) levantar o gênero dos autores; (3) levantar o local e sujeitos pesquisados; e (4) levantar os temas estudados por trabalhos teóricos/práticos e de pesquisa. 


\section{Método}

Por se tratar de uma pesquisa de levantamento foi estabelecido o seguinte percurso metodológico, que é descrito neste item.

Sujeitos-Juízes. Foram juízas duas psicólogas, professoras de Psicologia Escolar de uma Universidade Particular e doutorandas em Psicologia Escolar.

Material. Foram utilizados os Anais do I Congresso Nacional de Psicologia Escolar, publicado em 1992 pela ABRAPEE e PUCCAMP. Deste anal, foram apenas analisadas as sessões de comunicações científicas, da página 199 a 410, perfazendo o total de 22 sessões com 77 trabalhos publicados.

Procedimento. O material foi analisado independentemente pelos juízes, sendo elaboradas categorias de análise, nas quais poderiam ser registrados mais de um item. O índice de correlação, da análise de conteúdo das categorias, obtido entre os juízes foi de 0,87 , portanto, sendo significativo.

Tabela 1. Tipos de Pesquisas apresentadas nas sessões de comunicação do I Congresso de Psicologia Escolar distribuidos por Universidade Pública e Particular e por gênero

\begin{tabular}{|c|c|c|c|c|c|c|c|c|c|c|c|c|c|c|}
\hline Universo & \multicolumn{4}{|c|}{ Pública } & \multicolumn{4}{|c|}{ Particular } & \multicolumn{4}{|c|}{ Total } & \multirow{2}{*}{\multicolumn{2}{|c|}{$\begin{array}{l}\text { TOTAL } \\
\text { GERAL }\end{array}$}} \\
\hline Sexo & & 4 & & $\mathbf{F}$ & & $\mathbf{M}$ & & $\mathbf{F}$ & & M & & $\mathbf{F}$ & & \\
\hline in & $f$ & $\%$ & $f$ & 0 & & $\%$ & $f$ & $\%$ & & $\%$ & $\mathrm{f}$ & $\%$ & & $\%$ \\
\hline & & & & & 02 & 25,00 & & & 8 & 44,44 & 67 & & 9 & 3,40 \\
\hline & & & & & & & 07 & & & & 12 & & & ,55 \\
\hline & & 40 & & 3,85 & 02 & 25,00 & 11 & 5,94 & 06 & 33,33 & 14 & & 20 & 12,58 \\
\hline & & - & & & & - & 04 & 5,80 & 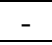 & - & 0,4 & 2,72 & 0,4 & 2,51 \\
\hline eórice & & - & & 28,20 & 04 & 50,00 & 28 & 40,58 & 04 & 22,22 & 50 & 34,01 & 54 & 33,96 \\
\hline tal & & 100 & 78 & 100,0 & 08 & 100,0 & 69 & 100,0 & 18 & 99,99 & 147 & 99,99 & 159 & 100, \\
\hline
\end{tabular}

\section{Resultados e Discussão}

Os resultados obtidos são apresentados na Tabela 1,2 e 3 sendo a primeira referente aos tipos de pesquisa, de universidade e de gênero de seus autores; a segunda refere-se ao tipo de trabalho apresentado nas sessões (teórico/prático ou de pesquisa), bem como a área de conhecimento, local e sujeitos estudados; e a terceira refere-se ao conteúdo e tema central enfocados.

Os dados da Tabela I revelaram que 43,40\% dos trabalhos apresentados são de pesquisas de levantamento, 33,96\% de trabalhos teóricos ou práticos, 12,58\% de pesquisas quasi-experimentais, $7,5 \%$ de pesquisas correlacionais e apenas de 2,51 \% de estudos experimentais. Estes resultados evidenciam que a maior parte da produção científica $(77,36 \%)$ da Psicologia Escolar, apresentada no I Congresso Nacional, vem de trabalhos que promovem apenas o crescimento horizontal da ciência, que fornecem informações mais descritivas, de suporte, de reflexão dos conhecimentos já existentes e de relatos de vivências na área escolar. Há uma minoria de trabalhos (2,51 \%) que promove o desenvolvimento vertical desta área de conhecimento. São estas pesquisas, as experimentais, que produzem resultados significantes sobre a relação das variáveis estudadas. As áreas de conhecimento mais desenvolvidas e cujo objeto de estudo é mais exata, como a Física ou a Matemática, produzem mais pesquisas experimentais promovendo o desenvolvimento e crescimento da sua ciência, tanto horizontal como verticalmente (Llagostera, 1991).

A Tabela 1 também apresenta a produção nas universidades públicas e particulares e do gênero masculino e feminino distribuídos pelo tipo de pesquisa. De forma geral, observa-se que a 
produção da universidade pública e particular, em termos de quantidade (respectivamente, $\mathrm{n}=88 \mathrm{e}$ 77), é basicamente a mesma, sendo que a diferença entre elas é de apenas onze trabalhos. Entretanto, ao analisar o tipo de pesquisa percebe-se que a universidade pública realiza $61,36 \%$ de pesquisas de levantamento contra $27,27 \%$ da particular; já a particular publica $41,56 \%$ de estudos teóricos/ práticos e a pública apresenta $25 \%$ deste tipo de produção; é importante destacar que apenas a universidade particular realiza trabalhos de pesquisa experimental (5,19\%). Estes dados refletem que a produção científica, na área da Psicologia Escolar, precisa realizar e desenvolver mais trabalhos com delineamento quasi-experimental e experimental para promover o avanço da ciência nesta área (Witter, 1977; Drew, 1980; Oakland e Wechsler, 1988; Oakland, 1989).

O gênero feminino apresenta uma produção superior $(n=147)$ ao gênero masculino ( $n=$ 18), reforçando os dados de pesquisas anteriores na área de Psicologia, como a de Witter e colaboradores (1992), que apresentou resultado semelhante ao investigar a produção científica em anais, resumos, periódicos, dissertações e teses.

Estes resultados evidenciam a necessidade de um maior empenho e esforço dos pesquisadores, das universidades, dos órgãos governamentais em investirem em pesquisas de delineamentos mais apurados e complexos que permitam uma melhor generalização dos dados obtidos, bem como, a verificação e determinação das relações entre as variáveis estudadas e investigadas nas pesquisas. Ao mesmo tempo, é importante no "saber-poder-fazer" da ciência, realizar pesquisas que contribuam tanto para a sociedade no geral como para a comunidade acadêmica.

A Tabela 2 apresenta os resultados quanto ao tema dos 1rabaJhos que versaram sobre quatro grandes categorias: Psicologia Escolar (32,43\%), Aluno (31,08\%), Professor (14,86\%) e Ensino/Educação (17,57\%). Os trabalhos de Witter (1987) e de Witter e colaboradores (1992), encontraram resultados semelhantes quando investigaram os sujeitos das pesquisas, em geral, na maioria dos trabalhos são investigadas variáveis referentes aos alunos e professores. 
Tabela 2. Tema, Local e Sujeitos estudados pelos trabalhos apresentados nas sessões de Comunicação

\begin{tabular}{|c|c|c|c|c|c|c|}
\hline \multirow{2}{*}{\begin{tabular}{l}
\multicolumn{1}{c}{ Trabalho } \\
Local e Sujeito \\
Tema
\end{tabular}} & \multicolumn{2}{|c|}{ Teórico-prático } & \multicolumn{2}{|c|}{ Pesquisa } & \multicolumn{2}{|c|}{ Total } \\
\hline & $\mathrm{f}$ & $\%$ & $\mathrm{f}$ & $\%$ & $\mathrm{f}$ & $\%$ \\
\hline $\begin{array}{l}\text { Psicologia } \\
\text { Escolar }\end{array}$ & 24 & 80,00 & 04 & 66,67 & 28 & 77,78 \\
\hline Educação & 05 & 16,67 & - & - & 05 & 13,89 \\
\hline $\begin{array}{l}\text { Análise de } \\
\text { Conteúdo }\end{array}$ & 01 & 3,33 & 02 & 33,33 & 03 & 8,33 \\
\hline Sub-total & $\begin{array}{c}30 \\
100,00\end{array}$ & & 06 & 100,0 & 36 & 100,0 \\
\hline \multicolumn{7}{|l|}{ Local } \\
\hline Pré-escola & - & - & 04 & 9,76 & 04 & 6,90 \\
\hline $1^{\circ} \mathrm{Grau}$ & 06 & 35,29 & 14 & 34,15 & 20 & 34,48 \\
\hline $2^{\circ} \mathrm{Grau}$ & - & - & 01 & 2,44 & 01 & 1,72 \\
\hline $3^{\circ} \mathrm{Grau}$ & 05 & 29,41 & 05 & 12,19 & 10 & 17,24 \\
\hline Vários - Misto & 03 & 17,65 & 09 & 21,95 & 12 & 20,69 \\
\hline Não especif. & 03 & 17,65 & 04 & 9,76 & 07 & 12,07 \\
\hline Outros & - & - & 04 & 9,76 & 04 & 6,90 \\
\hline Sub-total & $\begin{array}{c}17 \\
100,00\end{array}$ & & 41 & 100,01 & 58 & 100,0 \\
\hline \multicolumn{7}{|l|}{ Sujeitos } \\
\hline Aluno & 07 & 41,18 & 19 & 46,34 & 26 & 44,83 \\
\hline Professor & - & - & 04 & 9,76 & 04 & 6,90 \\
\hline Prof. - Aluno & 03 & 17,65 & 06 & 14,63 & 09 & 15,52 \\
\hline Vários - Misto & 05 & 29,41 & 03 & 7,32 & 08 & 13,79 \\
\hline $\begin{array}{l}\text { Psicólogo } \\
\text { Escolar }\end{array}$ & - & - & 05 & 12,19 & 05 & 8,62 \\
\hline Não especif. & 02 & 11,76 & 02 & 4,88 & 04 & 6,90 \\
\hline Outros & - & - & 02 & 4,88 & 02 & 3,45 \\
\hline Sub-total & $\begin{array}{c}17 \\
100,00\end{array}$ & & 41 & 100,00 & 58 & 100,01 \\
\hline
\end{tabular}

No que se refere ao local onde foram realizadas as pesquisas, as sessões de comunicações indicaram, praticamente a escola, sendo predominante os trabalhos desenvolvidos no $1^{\circ}$ grau (34,48\%), Misto (categoria que envolvia mais de um nível de escolaridade - 20,69\%), no $3^{\circ}$ grau (17,24\%), não especificado (12,07\%), pré-escola e outros $(6,9 \%)$ e $2^{\circ}$ grau $(1,72 \%)$. Os sujeitos levantados são: 44,83\% de alunos, 15,52\% de professores e alunos e 13,79\% de vários (esta categoria compreendeu equipe multidisciplinar, docentes e pessoal operacional e demais combinações do recurso humano existente na escola), as demais categorias apresentaram escores abaixo de 10\% (professores, psicólogo escolar, outros e não especificado). Estes resultados confirmam o exposto anteriormente, destacando que as pesquisas na área escolar se encontram no próprio ambiente escolar, e, conseqüentemente, no binômio aluno-professor.

A Tabela 3 apresenta dados, sobre o conteúdo ou tema central, que corroboram e destacam as mesmas variáveis, acrescentando apenas estudos sobre o Psicologia Escolar (32,43\%) devido à temática central do congresso que versava sobre a questão da identidade e perspectivas do profissional. Nesta categoria foram incluídos trabalhos sobre: associação, instituições, formação, 
atuação, função e papel do Psicólogia Escolar. As outras quatro categorias compreenderam os seguintes conteúdos e resultados: Aluno perfil, características, leitura e escrita, matemática, desenvolvimento humano, excepcional, comportamento e dificuldades, com 31,08\% do total dos dados; Professor - formação, atuação, função, interação com o aluno, metodologia, didática e comportamento (14,86\%); Ensino/Educação - problemas e distúrbios de aprendizagem, evasão escolar, integração universidade-comunidade, instrumento de avaliação, ensino-aprendizagem, prática pedagógica e adaptação escolar ( 17,57\%) e Outros - televisão, pais, teoria construtivista e informática (4,05\%).

Tabela 3. Conteúdo-Tema Central dos trabalhos teóricos-práticos e de pesquisa apresentados nas Sessões de Comunicação.

\begin{tabular}{|l|c|c|c|c|c|c|}
\hline \multicolumn{1}{|c|}{ Trabalho } & \multicolumn{2}{c|}{ Teórico-prático } & \multicolumn{2}{c|}{ Pesquisa } & \multicolumn{2}{c|}{ Total } \\
\hline $\begin{array}{l}\text { Conteúdo Tema } \\
\text { Central }\end{array}$ & $\mathrm{f}$ & $\%$ & $\mathrm{f}$ & $\%$ & $\mathrm{f}$ & $\%$ \\
\hline Psicologia Escolar & 36 & 60,00 & 12 & 13,64 & 48 & 32,43 \\
\hline Aluno & 11 & 18,33 & 35 & 39,77 & 46 & 31,08 \\
\hline Professor & 02 & 3,33 & 20 & 22,73 & 22 & 14,86 \\
\hline Ensino/Educação & 09 & 15,00 & 17 & 19,32 & 26 & 17,57 \\
\hline Outros & 02 & 3,33 & 04 & 4,54 & 06 & 4,05 \\
\hline \multicolumn{1}{|c|}{ Total } & 60 & 99,99 & 88 & 100,00 & 148 & 99,99 \\
\hline
\end{tabular}

Os dados desta pesquisa são preliminares. Entretanto, ressaltam a carência de pesquisas na área escolar, as quais estão limitadas inclusive quanto ao seu sujeito de estudo, enfatizam basicamente os alunos e professores. A área de conhecimento e de estudo da Psicologia Escolar é muito mais ampla, envolvendo não apenas o ambiente escolar como o familiar e a sociedade como um todo, logo, os sujeitos pesquisados devem ser ampliados, abrangendo pais, mães, diretores, profissionais da saúde, dirigentes de Estado, pessoal operacional e demais recursos humanos relacionados direta ou indiretamente à educação. A atuação, o ensino e a pesquisa, o trabalho profissional do psicólogo escolar têm desenvolvido-se nos últimos anos, se ampliado e incrementado, ao mesmo tempo, que se divulga e se propaga nas mais diversas direções, porém muitas têm sido as dificuldades e problemas para o desenvolvimento científico desta área, desde verbas até a falta de profissionalismo (Witter, 1977; Guzzo, 1987; Wechsler, 1987; e Witter, 1992).

Conclui-se que há necessidade de estudos mais amplos e com procedimentos mais sofisticados que contribuam para a criação de um banco de dados para a investigação do "estado da arte" na Psicologia Escolar no Brasil. Para tanto, é fundamental o trabalho conjunto dos pesquisadores e de instituições de ensino, de classe e governamentais que promovam a realização deste tipo de trabalho.

\section{Referências}

Conselho Federal de Psicologia (CFP) (1992). Psicólogo Brasileiro: construção de novos espaços. Organizadores: Ana Lúcia Francisco, Carolina de Rocio Klomfahs, Nádia Maria Dourado Rocha. Campinas, Átomo.

Drew, C. J. (1980). Introduction to designing and conducting research. St. Louis, The C. V. Mosby Comp. Eisenberg, N. (1991). Meta-analytic contributions to literature on prosocial behavior. Personality and Social Psychology Bulletin, 17(3): 273 - 282. 
Guzzo, R. S. L. (1987). Quem é o psicólogo escolar? Sua atuação prática. Anais da XVII Reunião Anual de Psicologia da SPRP. Ribeirão Preto: 186 - 190.

Llagostera, A. A. G. (1991). Produção técnico-científica do setor elétrico: estudo de seminários nacionais de produção e transmissão de energia elétrica na década de 1980. Dissertação de Mestrado, PUCCAMP.

Oakland, T. (1989). Psicologia escolar no Brasil: passado, presente e futuro. Psicologia: Teoria e Pesquisa, 5(2): $191-201$.

Oakland, T. e Wechesler, S. (1988). School Psychology in five South American contries: a 1989 perspective. Revista Interamericana de Psicologia, 22(1 e 2): 41 - 55.

Wechsler, S. M. (1987). A psicologia escolar no Brasil: dificuldades e possibilidades. Anais da XVII Reunião Anual de Psicologia da SPRP. Ribeirão Preto: 191 - 195.

Witter, G. P. (1977). O Psicólogo Escolar: pesquisa e ensino. Tese de Livre Docência, IPUSP, São Paulo.

Witter, G. P. (1987). Quem é o Psicólogo Escolar? Sua atuação prática. Anais da XVII Reunião Anual de Psicologia da SPRP. Ribeirão Preto: 183 - 185.

Witter, G. P. (1992). Psicologia Escolar: estudo de uma carreira/profissão. Anais do IV Encontro Paranaense de Psicologia. Curitiba: 1 - 6.

Witter, G. P. e col. (1992). Atuação do Psicólogo Escolar e Educacional no Brasil: perspectivas através de textos (1980 - 1992). In Conselho Federal de Psicologia. Psicólogo Brasileiro: construção de novos espaços. Campinas: Átomo. 